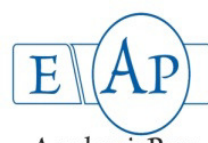

AcademicPres

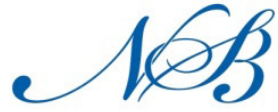

Notulae Scientia Biologicae

\title{
Host Vascular Plants for Pests in the Hydrographical Basin of the Otăsău River, Romania
}

\author{
Ion MITREA*, Nicolae Cristian FOTA
}

University ofCraiova, Faculty of Agriculture and Horticulture, 19 Libertătiii, 200583 Craiova, Romania;mitreaion@gmail.com (*orrespondingauthor)

\begin{abstract}
This study was carried out to investigate vascular host plant distribution in the hydrographical basin of the Otăsău River (Vâlcea County, in the Central Southern part of Romania). In order to determine the existing species in this area, fresh and dry material were collected. Specie identification was made based on a repetitive observation of the plants. A taxonomical aid was used to identify the species and classify them into different categories. In this research 368 taxa were investigated and registered as host vascular plants for pests from the studied area. The geoelement analysis shows that Eurasian, European, Central European, Cosmopolite and Circumpolar elements are predominant. Concerning the bioform spectrum the most representative components of the herbaceous layer are hemicryptophytes with 127 taxa. The study reveals that the studied area presents a high floristic diversity of the vascular plants (about 10\% of the Romanian flora).
\end{abstract}

Keywords: diversity, hemicryptophytes, pests, taxa, plant species

\section{Introduction}

The analysed territory is located in the middle part of Vâlcea County, in the Sub-Carpathian region of Oltenia, Romania; its northern limit is represented by the southern part of Buila Vânturarița National Park, while, in the south, is limited by Băbeni town, where the Otăsău River flows into the Bistrița River(Fig. 1).

The influence of the milder climate characteristic to Oltenia is quite obvious within the analysed territory, especially in the case of the southern slopes.

The hydrographical network is represented by the Otăsău River, which stems from Muntele Piatra/Stone Mountain (1581 $\mathrm{m})$; it gathers the streams from the southern slopes of Piatra and Albu Mountains (Bulzului Valley, Pătrunsa Valley) and flows from north-west to south-west and, then, it flows into the Bistrița River, within Frânceşti settlement. Its most important tributaries are the Bulzu and the Tisa streams; the confluence area being located in the proximity of Bărbăteşti settlement (Ujvari, 1972).

The high diversity of the relief is due to the altitude, orientation of the Sub-Carpathian summits which is generally from west to east showing an alternation of valleys and depressions, the presence of the Otăsău floodplain, the geomorphological particularities of all the relief forms, etc. (Fig. 2).

In the work entitled 'Short presentation of the flora of Romania', Grecescu (1898) mentioned some taxa from the region. Most of the mentioned taxa, with few exceptions, were also identified by us.

Scattered data on the flora and vegetation from the Otăsău basin also appear in certain papers elaborated by Buia and Păun
(1960); Popescu (1974); Popescu et al. (2001, 2003) However, all these studies make reference only to the mountain region.

In the neighbouring hydrographical basins, there have been made numerous studies of the flora and vegetation (Răduţoiu, 2008).

There have not been found any studies regarding the flora from the horticultural crops of this territory.

In Oltenia, studies regarding insect fauna have been carried out by Bobîrnac et al. (1973); Stănoiu and Năstase (1998); Chimişliu Cornelia (2000); Mitrea et al. (1998) etc.

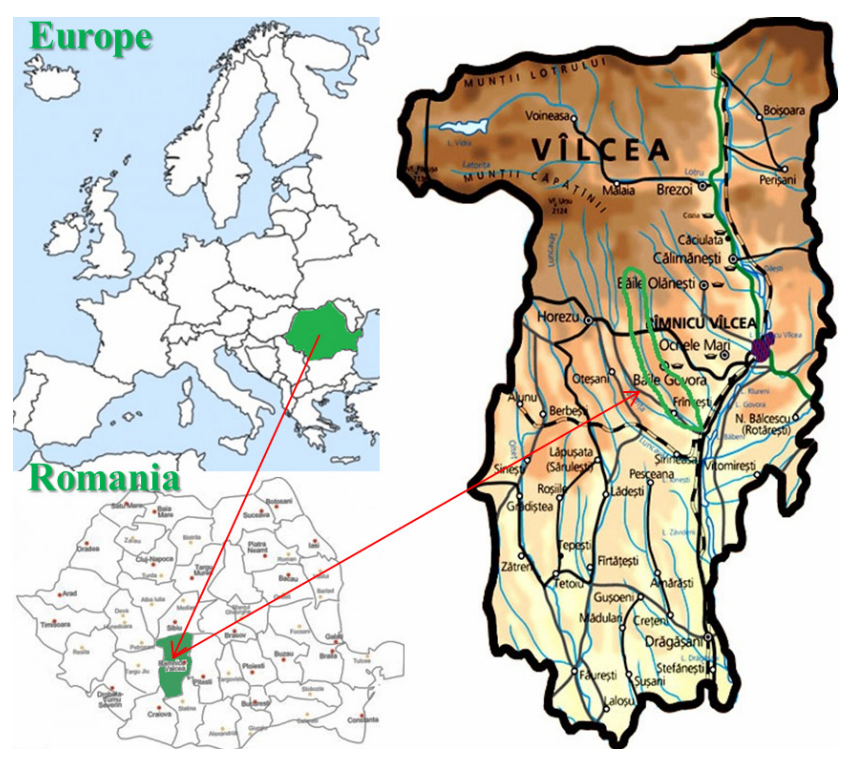

Fig. 1. Delimitation of the investigated area 


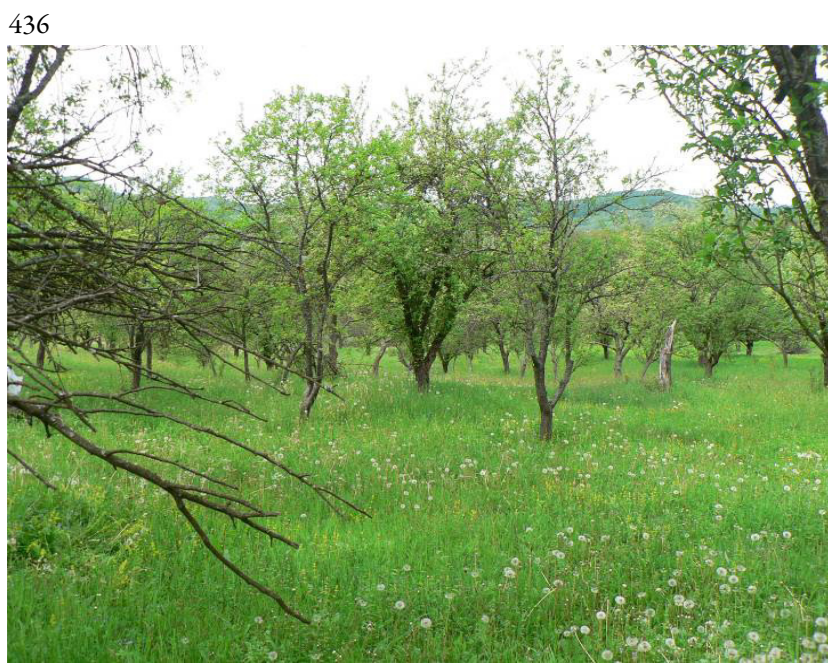

Fig. 2. General aspect from the investigated area

\section{Materials and Methods}

The first step in the research was to consult the scientific literature. Based on the documentation from this field, we conducted a personal repetitive research in order to observe the current situation of the flora and the interaction between the place and the pest agents.

The taxa were collected in bags in order to be immediately identified in the laboratory.

Taxa identification was made based on both dry preserved material and fresh material using recent sources of taxonomic information (Săvulescu, 1952-1976; Tutin et al., 1964-1980; Beldie, 1977, 1979; Rothmaler, 1994, 2002; Ciocârlan, 2000, 2009; Sârbu et al., 2013).

The author's abbreviations were made according to Brummitt and Powell (1992).

The families were mentioned in systematic order. For each family, genera and species were inserted in alphabetical order.

The determination of the entomological material was realised using the binocular loupe and different field guides from Panin (1957); Rogojanu and Perju (1979); Perju et al. (1983); Stănoiu and Năstase (1998), in the Entomology Laboratory of the Faculty of Agriculture and Horticulture from Craiova.

\section{Results and Discussions}

The research was carried out in the lower basin of the Otăsău River, that revealed the presence of numerous vascular plants (368 taxa) that are possible host species for the pests occured in the area. Their presentation is made on families, in systematic order, as follows: EQUISETACEAE: Equisetum arvense L. - G., Cosm.; E. palustre L. - G., Circ.; E. ramosissimum Desf. - G., Cosm.; E. telmateia Ehrh. - G., Circ.; MagnolioPHYTINA, MAGNOLIOPSIDA, ARISTOLOCHIACEAE: Asarum europaeum L. - G., Euras.; Aristolochia clematitis L. - G., Medit.; RANUNCULACEAE: Anemone nemorosa L. - G., Circ.; $A$. ranunculoides L. - G., Eur.; Caltha palustris L. - H., Circ.; Clematis vitalba L. - Ph., Eur. centr.; Consolida regalis S. F. Gray T., Eur.; Helleborus odorus Waldst. et Kit. - H., Balc.; R. arvensis L. - T., Euras.; R. auricomus L. - H., Euras.; R. ficaria L. subsp. bulbilifer Lambinon - H., Euras.; $R$ repens L. - H., Euras.; $R$. sardous Crantz - T., Eur.; $R$. sceleratus L. - T., Circ.;
PAPAVERACEAE: Chelidonium majus L. - H., Euras.; Papaver dubium L. subsp. dubium - T., Pont.-medit.; P. rhoeas L. - T., Cosm.; ULMACEAE: Ulmus glabra Huds. em Moss - Ph., Euras.; U. minor Mill. em Richens - Ph., Eur.; MORACEAE: Morus alba L. - Ph., China; CANNABACEAE (CANNABINACEAE): Humulus lupulus L. - H., Euras., Am. de N., URTICACEAE: Urtica dioica L. - T., Cosm.; U. urens L. - T., Cosm.; Parietaria officinalis L. - H., Medit.-submedit.; JUGLANDACEAE: Juglans regia L. - Ph., Centr. eur.-balc.cauc.; FAGACEAE: Fagus sylvatica subsp. moesiaca (Maly) Hjelmquist - Ph., Balc.; Quercus dalechampii Ten. - Ph., Medit.Carp.-Balc.; Q. polycarpa Schur - Ph., Carp.-Balc.-Cauc.; BETULACEAE: Alnus glutinosa (L.) Gaertn. - Ph., Euras.; PORTULACACEAE: Portulaca oleracea L. - T., Cosm. CARYOPHYLLACEAE: Arenaria serpyllifolia L. - T., Circ.; Cerastium brachypetalum Desp. ex Pers. - T., Centr.-eur.; $C$. semidecandrum L. - T., Eur.; Cucubalus baccifer L. - H., Euras.; Dianthus armeria L. - T., Eur.; D. carthusianorum L. - H., Eur.; Gypsophila muralis L. - T., Euras.; Lychnis coronaria (L.) Desr. H., Medit.; Myosoton aquaticum (L.) Moench - H., Euras.; Saponaria officinalis L. - H., Euras.; Silene latifolia Poir. subsp. alba (Mill.) Greuter et Burdet - T., H. Euras.; S. viridiflora L. H., Eur. centr.; S. vulgaris (Moench) Garcke - H., Euras.; Stellaria media (L.) Vill. - T., Cosm.; AMARANTHACEAE: Amaranthus albus L. - T., Adv. (Am. de N.); A. retroflexus L. - T., Adv. (Am. de N); CHENOPODIACEAE: *Atriplex hortensis L. - T., Adv. (As.); A patula L. - T., Circ.; Chenopodium album L. - T., Cosm.; POLYGONACEAE: Fallopia dumetorum L. - T., Circ.; Polygonum aviculare L. - T., Cosm.; P. minus Huds. - T., Eur. P. mite Schrank - T., Eur.; Rumex conglomeratus Murray H., Circ.; R. crispus L. - H., Euras.; R. pulcher L. - HT.-H., Centr. şi S. Eur.; $R$. sanguineus L. - H., Eur.; ROSACEAE: *Persica vulgaris Miller - Ph., China; Cerasus vulgaris Mill. - Ph., Eur. SE şi As. de V.; Crataegus monogyna Jacq. - Ph., Euras.; Fragaria vesca L. - H., Euras.; Geum urbanum L. - H., Circ.; *Malus domestica Borkh. - Ph., Potentilla argentea L. - H., Euras.; P. micrantha Ramond ex DC. - H., Centr. eur.-submedit.; P. reptans L. - H., Euras.; Prunus cerasifera Ehrh - Ph., Pont.-balc.; *P. domestica L. Ph., As. V.; P. spinosa L. - Ph., Eur.; Pyrus communis L. em. Gaertn. - Ph., Euras.; P. pyraster (L.) Burgsd. - Ph., Eur.; Rosa canina L. - Ph., Eur.; Rubus caesius L. - Ph., Eur.; $R$ candicans Weihe ex Rchb. subsp. thyrsanthus (Focke) Gayer - Ph., Eur. centr. and SV.; Sanguisorba minor Scop. - H., Euras.; Sorbus torminalis (L.) Crantz - Ph., Centr. eur.; FABACEAE: Amorpha fruticosa L. - Ph., North Am.; Coronilla varia L. - H, Centr. eur.submedit.; Dorycnium herbaceum Vill. - Ch, Eur. centr. and SE.; Galega officinalis L. - H, Pont.-medit.; Genista sagittalis (L.) Gams - Ch, Atl.-centr.-eur.-medit.; Genista tinctoria L. - Ch, Euras.; Lathyrus nissolia L. - T, Atl. medit.; L. sylvestris L. - H, Eur.; L. tuberosus L. - H, Euras.; L. venetus (Mill.) Wohlf. - G, Pont.-medit.; L. vernus (L.) Bernh. - G, Euras.; Lotus corniculatus L. - H, Euras.; Medicago lupulina L.- T.-H, Euras.; M. minima (L.) L. - T, Submedit.; ${ }^{*}$ M. sativa L. - Ch.-H, Medit.; Melilotus albus Medik. - HT, Euras.; M. officinalis Lam. - HT, Euras.; *Phaseolus vulgaris L. - T, South Am.; *Robinia pseudoacacia L. - Ph., North Am.; Trifolium arvense L. - T, Euras.; $T$. campestre Schreb. - T., Eur.; T. medium L. - H, Euras.; T. 
ochroleucon Huds. - H, Eur. centr.; T. pallidum Waldst. \& Kit. T.-HT, Medit.; T. striatum L. subsp. tenuiflorum (Ten.) Arcang. T., Atl.-medit.; Vicia angustifolia L subsp. segetalis (Thuill.) Arcang. - T., Euras.; V. cracca L. - H, Euras.; V. grandiflora Scop. - T., Pont.-balc.-cauc.; V. lathyroides L. - T.-HT, Eur. V. tetrasperma (L.) Schereb. - T, Euras.; $V$. villosa Roth - T.-HT, Eur.; LYTHRACEAE: Lythrum salicaria L. - H, Circ.; L. virgatum L. - H, Euras. cont.; ONAGRACEAE (OENOTHERACEAE): Circaea lutetiana L. - G, Euras-medit.; ELAEAGNACEAE: *Eleagnus angustifolia L. - Ph, As. temp.; CORNACEAE: Cornus mas L. - Ph, Pont.-medit; C. sanguinea L. - Ph, Eur. centr.; LORANTHACEAE: Loranthus europoeus Jacq. - Ep., Eur.; CELASTRACEAE: Evonymus europoeus L. - Ph, Eur.; EUPHORBIACEAE: Euphorbia amygdaloides L. - Ch, Centr.eur.-subatl-submedit.; E. cyparissias L. - H., Euras.; E. helioscopia L. T, Euras.; E. virgata Waldst. et Kit. - $\mathrm{H}$, Euras. cont.; VITACEAE: Vitis sylvestris C.C. Gmel. - Ph, Pont.-medit;; $V$. vinifera L. - Ph, As. SV. and medit. reg.; ACERACEAE: Acer campestre L. - Ph, Eur.; ${ }^{*}$. negundo L. - Ph, North Am.; $A$. platanoides L. - Ph, Eur.; A. tataricum L. - Ph, Euras. cont.; OXALIDACEAE: Oxalis corniculata L. - T.-H, Adv. (Medit.); GERANIACEAE: Erodium cicutarium (L.) L'Her. - T, Cosm.; Geranium molle L. - T, Euras.; G. pusillum L. - T, Eur.; ARALIACEAE: Hedera helix L. - Ph, Atl-medit.; APIACEAE (UMBELLIFERAE): Aegopodium podagraria L. - H.(G.), Euras.; *Anethum graveolens L. - T, SV As.; Anthriscus cerefolium (L.) Hoffm. subsp. trichosperma (Schult.) Arcang. - T, Pont.-medit.; *Apium graveolens L. - HT, Atl. Medit.; Berula erecta (Huds.) Coville - H.(HH.), Circ. Chaerophyllum aromaticum L. - H, Eur. centr.; C. temulum L. - HT, Eur.; Conium maculatum L. - HT, Euras.; Daucus carota L. subsp. carota - HT, Euras.; Eryngium campestre L. - H, Pont.-medit; Heradeum sphondylium L. - HT.H, Euras.; Oenanthe banatica Heuff. - H, Pont.-pan.-balc.; Pastinaca sativa L. subsp. urens (Req. ex Godron) Celak. - HT, Centr. Eur. Submedit.; Sium latifolium L. - HH, Euras.; Torilis arvensis (Huds.) Link - T, Eur. centr.; TILIACEAE: Tilia platyphyllos Scop. - Ph., Eur. centr.; T. tomentosa Moench - Ph, Balc.-pan.; MALVACEAE: Althaea officinalis L. - H., Euras. cont.; Hibiscus trionum L. - T., Euras.; Malva sylvestris L. - HT.-H., Euras.; VIOLACEAE: Viola alba Besser - H., Centr. eur. (submedit.); $V$. arvensis Murray - T., Cosm.; $V$. odorata L. - H., Atl-medit.; $V$. reichenbachiana Jordan ex Boreau - H., Euras.; BRASSICACEAE (CRUCIFERAE): Alliaria petiolata (Bieb.) Cavara et Grande - HT.-H., Euras.; Berteroa incana (L.) DC. HT., Euras.; Capsella bursa-pastoris (L.) Medik. - T.-HT., Cosm.; Cardaria draba (L.) Desv. - H., Euras. medit; Descurainia sophia (L.) Webb ex Prantl - T.-HT., Euras.; Erophila verna (L.) Chevall. - T., Euras.; Rorippa sylvestris (L.) Besser - H., Euras.; Sisymbrium officinale (L.) Scop.- T.-HT., Euras.; Thlaspi arvense L. - T.-HT., Euras.; T. perfoliatum L. - T.-HT., Euras.; SALICACEAE: Populus alba L. - Ph., Euras.; P. x canescens (Aiton) Sm. - Ph, Eur.; P. nigra L. - Ph., Euras.; Salix alba L. - Ph., Euras.; S. fragilis L. - Ph., Euras.; S. purpurea L. - Ph. Euras.; S. triandra L. - Ph., Euras.; PRIMULACEAE: Anagallis arvensis L. - T.-HT., Circ.; Lysimachia nummularia L. - Ch., Euras., North Am.; Primula acaulis (L.) L. - H., Submedit.; GENTIANACEAE: Centaurium erythraea Rafin. - T.-HT, Eur. centr.; ASCLEPIADACEAE:
Vincetoxicum hirundinaria Medik. - H., Euras cont. OLEACEAE: Fraxinus angustifolia Vahl. - Ph., Medit;; F. ornus L. - Ph., Submedit; Ligustrum vulgare L. - Ph., Eur. (submedit.); SOLANACEAE: Solanum dulcamara L. - Ch., Euras.; S. nigrum L. - T., Cosm.; CONVOLVULACEAE: Convolvulus arvensis L. (G.) H., Cosm.; BORAGINACEAE: Anchusa officinalis L. H.(HT.), Eur.; Cerinthe minor L. - T.-HT., Centr.-eur.-medit.; Cynoglossum officinale L. - HT., Euras. cont.; Echium vulgare L. HT., Euras.; Lappula squarrosa (Retz.) Dumort. - T., HT., Euras.; Lithospermum arvense L. - T., Euras.; L. purpurocaeruleum L. - H.G., Eur. centr.-submedit; Myosotis sparsiflora Mikan ex Pohl - T., Euras. cont.; Pulmonaria officinalis L. - H., Eur.; Symphytum officinale L. - H., Euras.; VERBENACEAE: Verbena officinalis L. H., Cosm.; LAMIACEAE (LABIATAE): Ajuga genevensis L. H., Euras.; A. reptans L.- H., Eur.; Ballota nigra L. - H., Eur. centr. şi NE.; Glechoma birsuta Waldst. et Kit. - H.(Ch.), Pont.-medit.centr.-eur.; Lamium amplexicaule L.- T., Euras.; L. purpureum L.T., Euras.; Leonurus cardiaca L. - H., Euras.; Lycopus europoeus L. H.(HH.), Euras.; L. exaltatus L. - H.(HH.), Euras. cont;: Mentha arvensis L. - H.(G.), Circ.; M. longifolia (L.) Huds. - H., Euras.; $M$. pulegium L. - H., Euras. (submedit.); Prunella laciniata (L.) L. - H., Centr. eur.-medit.; P. vulgaris L. - H. Cosm.; Salvia glutinosa L. H., Euras. (mont.); S. nemorosa L. - H., Pont--medit-centr.eur.; $S$. verticillata L. - H., Centr.-eur.-medit.; Teucrium chamoedrys L. Ch., Eur. centr. (submedit); T. pannonicus All. subsp. auctus (Lyka) Soo - Ch., Euras. cont:; T. pulegioides L. - Ch., Eur. (mont.); PLANTAGINACEAE: Plantago lanceolata L. - H., Euras.; $P$. major L. - H., Euras.; P. scabra Moench - T., Cont. euras.; SCROPHULARIACEAE: Gratiola officinalis L. - H., Circ; Kicksia elatine (L.) Dumort. subsp. sieberi (Arcang.) Hayek - T., Eur. de S.; Linaria genistifolia (L.) Mill. - H., Cont. euras.; $L$. vulgaris Mill. - H., Euras.; Melampyrum arvense L. - T., Eur.; Odontites vernus (Bellardi) Dumort. subsp. serotinus (Dumort.) Corb. - T., Euras.; Verbascum phlomoides L. - HT., Eur. centr. and SE; Veronica anagallis-aquatica L. - H.(HH.), Circ; $V$. chamaedrys L. subsp. chamaedrys - H.-Ch., Euras.; V. hederifolia L. - T., Euras.; $V$.persica Poir. - T., Adv.(SV. As.); $V$.polita Fr. - T., Euras.; $V$. triphyllos L. - T., Eur.; CAMPANULACEAE: Campanula glomerata L. - H., Euras.; C. rapunculoides L. - H., Euras.; RUBIACEAE: Asperula taurina L. subsp. leucanthera (G. Beck) Hayek - H., Medit; Cruciata glabra (L.) Ehrend. - H., Euras.; C. laevipes Opiz - H., Euras.; Galium aparine L. - T., Circ.; G. mollugo L. - H., Euras.; G. odoratum (L.) Scop. G., Euras.; $G$. verum L.- H., Euras.; Sherardia arvensis L. - T., Euras.; CAPRIFOLIACEAE: Sambucus ebulus L. - H., Euras. (submedit.); S. nigra L. - Ph., Eur.; VALERIANACEAE: Valeriana officinalis L. - H., Euras. (submedit.); Valerianella locusta (L.) Laterrade - T., Eur.; DIPSACACEAE: Dipsacus fullonum L. HT., Submedit;; D. laciniatus L. - HT., Euras. cont.; Knautia arvensis (L.) Coult. - H., Eur.; Scabiosa ochrolenca L. - HT.-H., Euras. cont.; ASTERACEAE (COMPOSITAE); Tubuliflorae (Asteroideae): Acbillea millefolium L. - H., Euras. Ambrosia artemisiifolia L. - T., Adv. (North Am.); Anthemis austriaca Jacq. T., Centr.-eur.-pont.; Arctium lappa L. - HT., Euras.; Artemisia absinthium L. - H.(Ch.), Euras.; A. vulgaris L. - H., Circ.; Bellis perennis L. - H., Eur.; Bidens tripartita L. - T., Euras.; Carduus acanthoides L. - HT., Eur.; C. vulgaris L. - HT., Euras.; 
438

Carthamus lanatus L. - T., Pont.-medit.; Centaurea apiculata Ledeb. subsp.spinulosa (Rochel) Dostal - H., Centr. şi SE. Eur.; $C$. biebersteinii DC. - HT.-H., Pont.-pan.-balc.; C. cyanus L. - T.HT., Medit. today Cosm.; $C$. iberica Trev. - HT., Pont.-balc.; $C$. solstitialis L. - HT., Medit.; C. stenolepis A. Kern. - H., Centr. and SE. Eur.; Chondrilla juncea L. - HT.-H., Cont. euras.; Cichorium intybus L. - H., Euras.; Cirsium arvense (L.) Scop. - G., Euras.; $C$. vulgare (Savi) Ten. - HT., Euras.; Conyza canadensis (L.) Cronq. T., Adv. (Am. de N.); Erigeron ánnuus (L.) Pers. - T., HT., H., Adv. (North Am.); Eupatorium cannabinum L. - H., Euras.; Filago vulgaris Lam. - T., Euras. de S.; Galinsoga ciliata (Rafin.) Bake - T., Adv. (South Am.); G.parviflora Cav. - T., Adv. (South Am.-Peru), today Cosm; Inula britannica L. - HT., Euras.; Leontodon autumnalis L. - H., Euras.; Leucanthemum vulgare Lam. - H., Euras.; Matricaria perforata Merat - T.-HT., Euras.; Mycelis muralis (L.) Dumort. - H., Eur.; Onopordum acanthium L. HT., Euras.; Pulicaria dysenterica (L.) Bernh. - H., Centr. eur.; $P$. vulgaris Gaertn. - T., Euras.; Senecio erucifolius L. - H., Euras.; $S$. vernalis Waldst. et Kit. - T., Euras. cont.; S. vulgaris L. - T., Euras.; Solidago virgaurea L. - H., Circ.; Sonchus arvensis L. - G., Euras.; Tanacetum corymbosum (L.) Schultz Bip. - H., Euras.; T. vulgare L. - H., Euras.; Taraxacum officinale Web. ex Wiggers - H., Euras.; Tragopogon pratensis L. subsp. orientalis (L.) Čelak. - HT.-H., Eur. centr. and E.; Tussilago farfara L. - G., Euras.; Xanthium italicum Moretti-T., South Eur. X. spinosum L. - T., Adv. (Am. de S.) today Cosm.; Xeranthemum annuum L. - T., Pont-medit; X. cylindraceum Sibt. et Sm. - T., Pont.-medit;; Liguliflorae (Cichorioideae): Cichorium intybus L. - H., Euras.; Crépis biénnis L. - HT., Eur.; Hieracium sabaudum L. - H., Eur.; Liliopsida (Monocotyledonatae), LILIACEAE: Asparagus tenuifolius Lam. G., Pont.-medit;; Colchicum autumnale L. - G., Centr. eur.; Erythronium dens-canis L. subsp. niveum (Baumg.) Buia et Păun G. V. and SV. Rom. End.; Gagea lutea (L.) Ker.-Gawl. - G., Euras.; Muscari comosum (L.) Mill. - G., Eur. (except N.); Ornithogalum divergens Boreau - G., Medit.; O. pyramidale L. - G., Centr. eur.; Polygonatum latifolium (Jacq.) Desf. - G., Pont.-pan.-balc.; ALLIACEAE: Allium ursinum L. subsp. ucrainicum Kleopow et Oxner - G., Pont--medit; $A$. vineale L. - G., Eur.; IRIDACEAE: Iris psendacorus L. - G., Eur.; JUNCACEAE: Juncus articulatus L. - H., Circ.; J. effusus L. - H., Cosm.; J. inflexus L.- H., Euras.; Luzula campestris (L.) DC. - H., Circ.; CYPERACEAE: Bolboschoenus maritimus (L.) Palla G.(HH.), Cosm.; Carex hirta L.- G., Circ.; C. praecox Schreb. - G., Euras. cont.; C. remota L. H., Circ.; C. riparia Curtis - G.(HH.), Euras.; Cyperus fuscus L. T., Euras.; Eleocharis palustris (L.) Roemer et Schultes - G.(HH.), Cosm.; Schoenoplectus tabernaemontani (C.C. Gmelin) Palla G.(HH.), Euras.; Scirpus sylvaticus L. - G., Circ.; POACEAE (GRAMINEAE): Aegilops cylindrica Host - T., Cont. euras.; Agrostis capillaris L. - H.(G.), Circ.; Apera spica-venti (L.) Beauv. T., Euras.; Brachypodium sylvaticum (Huds.) Beauv. - H., Euras. (submedit.); Bromus arvensis L. - T.-HT., Euras.(submedit.); $B$. inermis Leysser - $\mathrm{H}$., Cont. euras.; B. sterilis L. - T., Euras.(submedit.); B. tectorum L. - T., Euras. cont.; Calamagrostis epigeios (L.) Roth - G., Euras.; Cynodon dactylon (L.) Pers. - G., Cosm.; Dactylis glomerata L. - H., Euras.; Dasypyrum villosum (L.) P. Candargy - T., Medit.; Dichanthium ischaemum (L.) Roberty - H., Euras. (submedit.); Digitaria sanguinalis (L.) Scop

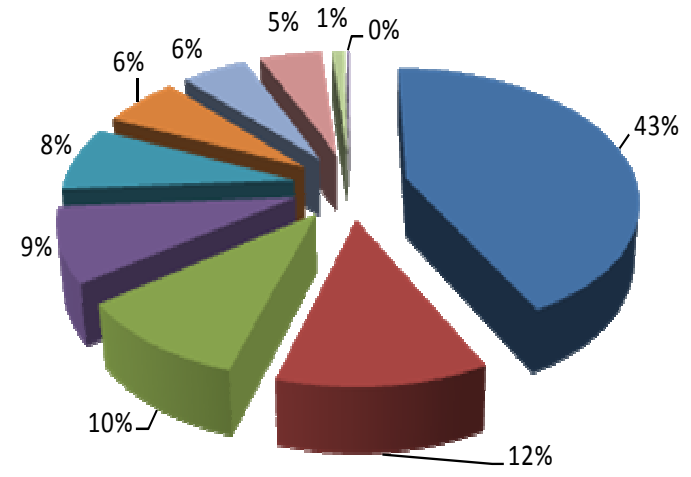

Euras $\square$ Eur $\square$ Centr. Eur. $\square$ Cosm $\square$ Circ $\square$ Medit $\square$ Pont $\square$ Adv $\square$ Balc $\square$ End

Fig. 3. Geoelement spectrum

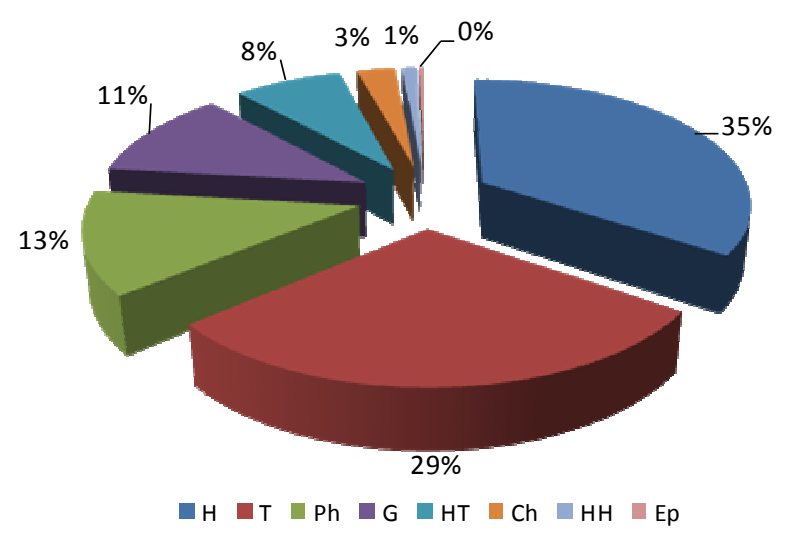

Fig. 4. Bioform spectrum

- T., Cosm.; Echinochloa crus-galli (L.) Beauv. - T., Cosm.; Eragrostis minor Host - T., Centr. eur.-medit;; Festuca gigantea (L.)Vill. - H., Euras.; F. heterophylla Lam. - H., Centr. eur.submedit; F. pratensis Huds. - H., Euras.; F. valesiaca Schleicher ex Gaudin - H., Cont. euras.; Glyceria notata Chevall. H. (HH.) Circ.; Hordeum murinum L. - T., Euras.; Leersia oryzoides (L.) Swartz - G. (HH.), Circ.; Lolium perenne L. - H., Cosm.; Melica uniflora Retz. - H.(G.), Centr. eur.-submedit; Phragmites australis (Cav.) Steudel - G.(HH.), Cosm.; Poa angustifolia L. - H. Euras.; P. annua L. - T.-H., Cosm.; P. palustris L. - H., Circ.; P. pratensis L. - H., Circ. (today Cosm.); P. sylvicola Guss. - H., Submedit.; P. trivialis L. - H., Euras.; Sclerochloa dura (L.) Beauv. - T., Medit.; Setaria pumila (Poir.) Schultes - T., Cosm.; S. viridis (L.) Beauv. T., Euras., today Cosm.; Sorghum halepense (L.) Pers. - G., Medit;; SPARGANIACEAE: Sparganium erectum L. - G.(HH.), Euras.; TYPHACEAE: Typha angustifolia L. - G.(HH.), Circ.; $T$. latifolia L. - G.(HH.), Cosm.; ARACEAE: Arum orientale Bieb. G. V, Centr-eur-medit. LEMNACEAE: Lemna minor L. HH., Cosm.

The flora list from the Otăsău hydrographic basin comprises 368 taxa distributed as it follows: 4 - Pteridophyta and 364 Spermatophyta.

According to the analysis of the geo-elements, it can be noticed the predominance of Eurasian elements, followed by the European, Central European, Cosmopolite and Circumpolar ones. An important number of species have Pontic, Baltic and Sub-Mediterranean origins (Fig. 3). 
Table 1. Entomofauna collected from the hydrographical basin of the Otăsau River according to the host plant

\begin{tabular}{|c|c|c|}
\hline No. & Collected entomofauna & Host plant \\
\hline 1 & Eriophyestristriatus Nalepa & Juglansregia L. \\
\hline 2 & Myzodesperisicae Sulzer; Anarsialineatella L. & Persica vulgaris Mill. \\
\hline 3 & Myzus cerasi L:; Epicometis hirta L.; Rhagoletiscerasi L. & Cerasus vulgaris Mill. \\
\hline 4 & Myzuscerasi L; Rhagoletiscerasi L. & Cerasus avium $\mathrm{L}$. \\
\hline 5 & $\begin{array}{l}\text { Epicometis hirta L:; Melolontha melolontha L:; Agriotes spp.L. pe Fragaria ananassa; Aphispomi De Geer; Quadraspisiotusperniciosus } \\
\text { Comstock; Anthonomuspomorum L:; Epicometishirta L.; Cydiapomonella L. }\end{array}$ & Malus domestica Borkh. \\
\hline 6 & Hyalopteruspruni Geoffroy; Quadraspisiotusperniciosus Comstock;Melolontha melolontha L., Cetonia aurata L. & Prunuscerasifera Ehrh. \\
\hline 7 & Hyalopteruspruni Geoffroy; Quadraspisiotuspermiciosus Comstock; Epicometishirta L; Cydiafunebrana L.; Eurytoma schreineri Schr. & Prunusdomestica Ehrh. \\
\hline 8 & Anthonomusrubi Herbst:; Myzusfragariae Theob; Epicometishirta L:; Melolonthamelolontha L:; Agriotes spp.L. & $\begin{array}{l}\text { Fragaria x ananassa (Duchesne) Decne. } \\
\text { et Naudin }\end{array}$ \\
\hline 9 & Eriophyesvitis L: Lobesia botrana Den \& Schiff; Clysia ambiguella $\mathrm{Hb}$. & Vitisvinifera $\mathrm{L}$ \\
\hline 10 & Acanthoscelidesobsoletus L; & Phaseolusvulgaris $\mathrm{L}$. \\
\hline 11 & Hyphantriacunea L. & Morusalba L. \\
\hline 12 & Agrolimaxagrestis L. & Atriplexhortensis L. \\
\hline 13 & $\begin{array}{l}\text { Graphosoma lineatum } \mathrm{L} \text {.; Melolontha melolontha } \mathrm{L} \text {., Cetonia aurata L. on Anethum graveolens; Grillotalpa grillotalpa L:; Graphosoma } \\
\text { lineatum L.; Melolontha melolontha L.; Epicometishirta L. on Apium graveolens and Scoliaflavifrons L.; Xilocopa violaceae L. }\end{array}$ & Bellisperennis L. \\
\hline
\end{tabular}

With regard to the analysis of bioforms, the first position is occupied by hemicryptophytes with 127 taxa (35\%). They represent the main components of the herbaceous layer (Fig. 4).

In Table 1 entomofauna is rendered which was collected from the Otăsau basin. Its grouping was made according to the place where the host plant was found.

\section{Conclusions}

The pest identification form this territory indicates the floristic diversity of this basin. There was registered a higher frequency in the case of the plants cultivated by local people in this region. Among the identified species that are hosts for animal pests we render the most popular ones found in the basin of Otăsău River: Hyphantria cunea L. on Morus alba L; Eriophyes tristriatus Nalepa on Juglans regia L.; Agrolimax agrestis L. on Atriplex hortensis L.; Myzodes perisicae Sulzer; Anarsia lineatella L. on Persica vulgaris Mill.; Myzus cerasi L.; Epicometis hirta L.; Rhagoletis cerasi L. on Cerasus vulgaris Mill.; Myzus cerasi L.; Rhagoletis cerasi L. on Cerasus avium L.; Anthonomus rubi Herbst.; Myzus fragariae Theob; Epicometis hirta L; Melolontha melolontha L.; Agriotes spp. L. on Fragaria x ananassa (Duchesne) Decne. et Naudin; Aphis pomi de Geer; Quadraspisiotus perniciosus Comstock; Anthonomus pomorum L.; Epicometis hirta L.; Cydia pomonella L. on Malus domestica L.; Hyalopterus pruni Geoffroy; Quadraspisiotus perniciosus Comstock; Melolontha melolontha $\mathrm{L}$., Cetonia aurata $\mathrm{L}$. on Prunus cerasifera L.; Hyalopterus pruni Geoffroy; Quadraspisiotus perniciosus Comstock; Epicometis hirta L.; Cydia funebrana L; Eurytoma schreineri Schr. on Prunus domestica L.; Acanthoscelides obsoletus L; on Phaseolus vulgaris L.; Eriophyes vitis L., Lobesia botrana Den \& Schiff; Chysia ambiguella $\mathrm{Hb}$. on Vitis vinifera L.; Graphosoma lineatum L.; Melolontha melolontha L., Cetonia aurata L. on Anethum graveolens; Grillotalpa grillotalpa L; Graphosoma lineatum L.; Melolontha melolontha L.; Epicometis hirta L. on Apium graveolens L. and Scolia flavifrons L.; Xilocopa violaceae $\mathrm{L}$. on Bellisperennis $\mathrm{L}$.

\section{References}

Beldie A (1977, 1979). Flora României. Determinator ilustrat al plantelor vasculare. Ed Academiei Române, Bucureşti Vol I-II, pp $406 ; 412$.
Bobîrnac B, Matei I, Costescu C (1973). Entomofauna. In: Pajșșile din zona subcarpatică a Olteniei. Ed Scrisul Românesc, Craiova pp 63-82.

Brummitt RK, Powell CE (1992). Authors of plant names. Royal Botanic Gardens, Kew.

Buia A, Păun M (1960). Plante noi şi rare din Oltenia. Contribuții Botanice pp 141-148.

Chimişliu C (2000). Contribuții la cunoaşterea subfamiliilor Coprinae şi Geotrupinae (Insecta: Coleoptera: Scarabaeidae) din fauna de coleoptere a Olteniei, Muzeul Olteniei Craiova. Studii şi comunicări Vol XXI pp 87-90.

Ciocârlan V (2000). Flora ilustrată a României. Pteridophyta et Spermatophyta. Ed Ceres, Bucureşti.

Ciocârlan V (2009). Flora ilustrată a României. Pteridophyta et Spermatophyta. Ed Ceres, Bucureşti.

Grecescu D (1898). Conspectul Florei României. Bucureşti.

Mitrea I, Bobîrnac B, Mitrea R (1998). Entomofauna unor culturi legumicole din zona subcarpatică a Olteniei. Lucrări Științifice Seria Horticultură, Univ Agron Med Vet "Ion Ionescu de la Brad", Iaşi Vol 41 pp 352-357.

Panin S, Săvulescu N (1957). Fauna Republicii Populare Romane Insecta Coleoptera. Ed Academiei Române, Bucuresti.

Perju T, Bobârnac B, Costescu C, Duvlea I, Filipescu C, Ghizdavu I, Paşol P (1983). Entomologie agricolă. Ed Didactică și Pedagogică Bucureşti pp 154-477.

Popescu G (1974). Studiul floristic şi geobotanic al Bazinului hidrografic al Bistriței-Vîlcii. Teza de doctorat, București.

Popescu G, Costache I, Răduțoiu D, Boruz V (2003). Valea Rea Radovan, Dolj district, floristic and vegetation point of great scientific importance. Acta Horti Botanici Bucurestiensis 30:8394.

Popescu G, Costache I, Răduțoiu D, Gămăneci G (2001). Vegetația pajiştilor (Cap 4). In: Ionescu I (Ed). Pajiştile permanente din nordul Olteniei. Ed Universitaria, Craiova pp 116-215.

Răduțoiu D (2008). Flora și vegetația Bazinului Cernei de Olteț. Ed Sitech, Craiova.

Rogojanu V, Perju T (1979). Determinator pentru recunoaşterea dăunătorilor plantelor cultivate. Ed Ceres, Bucureşti. 
440

Rothmaler W (1994). Excursionsflora von Deutschland-Band 48 Anflage. Gustav Fischer VerlagJena-Stuttgart.

Rothmaler W (2002). Exkursionsflora von Deutschland Gefabpflanzen: Kritischer Band 4. Spektrum Akademischer Verlag Heidelberg Berlin.

Sanda V (1980). Cenotaxonomia şi corologia grupărilor vegetale din România. Stud Com 24, Supliment Ştiințele Naturii Muzeul Brukenthal, Sibiu.

Sanda V (2002). Vademecum cenostructural privind covorul vegetal din România. Ed Vergiliu, București.

Sanda V, Popescu A, Stancu DI (2001). Structura cenotică şi caracterizarea ecologică a fitocenozelor din România. Ed Conphis, Bucureşti.
Sârbu I, Ştefan N, Oprea A (2013). Plante vasculare din România Determinator ilustrat de teren. Ed Victor B Victor, Bucureşti. Săvulescu T (1952-1976). Flora RPR-RSR. Ed Academiei Române, Bucureşti Vol I-XIII.

Stănoiu I, Năstase A (1998). Insecte din Romania. Ed Dova, Craiova.

Tutin TG, Heywood VH, Burges NA, Moore DM, Valentine DH, Walters SM, Webb DA (1964-1980). Flora Europaea. Vols I-V. The University Press, Cambridge.

Ujvari I (1972). Geografia apelor României. Ed Științifică, București. 